PROCEEDINGS OF THE AMERICAN MATHEMATICAL SOCIETY

Volume 124, Number 8, August 1996

\title{
AFFINE MAPPINGS OF INVERTIBLE OPERATORS
}

\author{
LAWRENCE A. HARRIS AND RICHARD V. KADISON
}

(Communicated by Palle E. T. Jorgensen)

\begin{abstract}
The infinite-dimensional analogues of the classical general linear group appear as groups of invertible elements of Banach algebras. Mappings of these groups onto themselves that extend to affine mappings of the ambient Banach algebra are shown to be linear exactly when the Banach algebra is semi-simple. The form of such linear mappings is studied when the Banach algebra is a $\mathrm{C}^{*}$-algebra.
\end{abstract}

\section{INTRODUCTION}

In this note, we study affine mappings between Banach algebras that carry distinguished sets of elements - notably, the set of invertible elements and the set of singular elements - in one Banach algebra into and onto the corresponding set in the other algebra. In Section 2, we consider the "onto" case and prove that such mappings are always linear if and only if the Banach algebra is semi-simple (Proposition 2.1). As a corollary (2.4), we show that $A+T$ has spectrum contained in the spectrum of $B+T$ for all $T$ in a $C^{*}$-algebra, where $A$ and $B$ lie in that algebra, if and only if $A=B$.

In Section 3, we examine the linear case of such mappings. We conjecture their form for $\mathrm{C}^{*}$-algebras and prove some results supporting that conjecture. A notion of "spectral additivity" is introduced that describes the resulting spectra when a given element of a $\mathrm{C}^{*}$-algebra is added to each other element of the algebra. Central elements are characterized in terms of this spectral effect in [4].

In Section 4, we establish the necessary and sufficient conditions for affine mappings on a $\mathrm{C}^{*}$-algebra of the form $Z \rightarrow A_{1} Z A_{2}+B$ to carry the sets of invertible or singular elements in a $\mathrm{C}^{*}$-algebra into themselves.

We establish some notation that is used throughout. Let $X$ be a complex normed linear space, and let $\mathfrak{A}$ be a complex subspace of a complex Banach algebra with identity $e$ such that $\mathfrak{A}$ contains $e$. Define

$$
\begin{aligned}
\mathfrak{A}_{\text {inv }} & =\{z \in \mathfrak{A}: z \text { is invertible }\}, \\
\mathfrak{A}_{\exp } & =\{\exp (z): z \in \mathfrak{A}\}, \\
\mathfrak{A}_{\text {qnil }} & =\{z \in \mathfrak{A}: e+\lambda z \text { is invertible for all } \lambda \text { in } \mathbb{C}\}, \\
\mathfrak{A}_{\text {rad }} & =\{z \in \mathfrak{A}: e+z x \text { is invertible for all } x \text { in } \mathfrak{A}\} .
\end{aligned}
$$

Received by the editors February 13, 1995.

1991 Mathematics Subject Classification. Primary 46L05.

Key words and phrases. Banach algebra, $\mathrm{C}^{*}$-algebra, invertible elements.

(C)1996 American Mathematical Society 
The set $\mathfrak{A}_{\text {qnil }}$ is the set of all quasi-nilpotent elements in $\mathfrak{A}$. When $\mathfrak{A}$ is an algebra, $\mathfrak{A}_{\text {rad }}$ is the Jacobson radical of $\mathfrak{A}$ and $\mathfrak{A}$ is said to be semi-simple when $\mathfrak{A}_{\text {rad }}$ is $\{0\}$. The Banach algebra $\mathcal{B}(X)$ of all bounded linear transformations of $X$ into itself is semi-simple. (For an account of this, see [5, p. 702].) Each $\mathrm{C}^{*}$-algebra is semi-simple. To see this, note that if $z$ is in the radical of a $\mathrm{C}^{*}$-algebra, then so is $z z^{*}$; whence $\lambda e+z z^{*}$ is invertible for all non-zero $\lambda$ in $\mathbb{C}$. Since $z z^{*}$ is self-adjoint with spectrum $\{0\}$, it follows that $z z^{*}=0$ and hence $z=0$.

Let $\mathfrak{A}_{\text {inv }}^{\mathrm{e}}$ denote the identity component of $\mathfrak{A}_{\text {inv }}$ and $\mathfrak{A}_{\text {sing }}$ denote $\mathfrak{A} \backslash \mathfrak{A}_{\text {inv }}$. Then

$$
\mathfrak{A}_{\text {inv }}^{\mathrm{e}} \subseteq \mathfrak{A}_{\text {inv }}, \quad \mathfrak{A}_{\text {rad }} \subseteq \mathfrak{A}_{\text {qnil }} \subseteq \mathfrak{A}_{\text {sing }} .
$$

Also, $\mathfrak{A}_{\exp } \subseteq \mathfrak{A}_{\text {inv }}^{\mathrm{e}}$ when $\mathfrak{A}$ is a Banach algebra.

\section{SuRJeCtive AFFine MAPPINGS}

A mapping $\phi$ of $X$ into $\mathfrak{A}$ is affine when there are a $b$ in $\mathfrak{A}$ and a linear mapping $L$ of $X$ into $\mathfrak{A}$ such that $\phi(x)=L(x)+b$ for each $x$ in $X$. We begin with some basic observations about affine mappings that take a set of invertible or singular elements onto itself.

Proposition 2.1. Let $\mathfrak{A}$ be a complex Banach algebra with identity, and let $T$ be any one of the sets $\mathfrak{A}_{\mathrm{inv}}^{\mathrm{e}}, \mathfrak{A}_{\mathrm{inv}}, \mathfrak{A}_{\mathrm{qnil}}$ or $\mathfrak{A}_{\mathrm{sing}}$. Then every affine mapping of $\mathfrak{A}$ into $\mathfrak{A}$ that maps $T$ onto itself is linear if and only if $\mathfrak{A}$ is semi-simple.

Proposition 2.2. Let $\mathfrak{A}$ be a complex subspace of $\mathcal{B}(\mathcal{H})$ containing the identity operator I on $\mathcal{H}$, where $\mathcal{H}$ is a complex Hilbert space. If $T$ is a subset of $\mathfrak{A}_{\mathrm{inv}}$ such that $-T \subseteq T, T^{*} \subseteq T$ and $\lambda I \in T$ for all non-zero complex $\lambda$, then every affine mapping of $\mathfrak{A}$ into $\mathcal{B}(\mathcal{H})$ that maps $T$ or $\mathfrak{A} \backslash T$ onto itself is linear.

If $\mathfrak{A}$ contains the adjoints of each of its elements, then the hypotheses of Proposition 2.2 hold when $T$ is $\mathfrak{A}_{\text {inv }}$ or $\mathfrak{A}_{\text {inv }}^{\mathrm{e}}$ and when $\mathfrak{A} \backslash T$ is $\mathfrak{A}_{\text {sing }}$ or $\mathfrak{A}_{\text {qnil }}$. If $\mathfrak{A}$ is a $\mathrm{C}^{*}$-algebra with identity, the hypotheses also hold when $T=\mathfrak{A}_{\text {exp }}$. The following simple lemma allows us to reduce to the case where our affine mapping is a translation.

Lemma 2.3. Let $T$ be a subset of a normed linear space $X$, and suppose that $\phi$ is an affine mapping of $X$ into itself such that $\phi(T)=T$. If $-T \subseteq T$, then $y-2 \phi(0) \in T$ whenever $y \in T$.

Proofs. To prove Lemma 2.3, note that, given $y$ in $T$, there is an $x$ in $T$ such that $\phi(x)=y$. Expressing $\phi$ as the sum of a linear mapping and a translation, we have that $y-2 \phi(0)=-\phi(-x) \in T$.

To prove Proposition 2.1, let $\phi$ be an affine mapping of $\mathfrak{A}$ into $\mathfrak{A}$ such that $\phi(T)=T$. We want to show that $\phi(0)=0$. The mapping $y \rightarrow y-2 \phi(0)$ of $\mathfrak{A}$ onto $\mathfrak{A}$ is affine, and by Lemma 2.3 this mapping carries $T$ into $T$. Thus we need to consider only mappings $\phi$ of the form $\phi(z)=z+b$ for which $\phi(T) \subseteq T$. Suppose $T=\mathfrak{A}_{\mathrm{inv}}$ or $T=\mathfrak{A}_{\mathrm{inv}}^{\mathrm{e}}$. Given $x$ in $\mathfrak{A}$, choose a positive $t$ such that $t\|x\|<1$ and let

$$
y=(t e+b)(t x-e)^{-1} .
$$

Then $y \in T$ since $e-t x \in T$ and $t e+b \in T$. Hence $y+b \in T$ and $(y+b)(t x-e)=$ $t(e+b x)$, so that $e+b x$ is invertible. Therefore, $b \in \mathfrak{A}_{\mathrm{rad}}$.

Now suppose that $T=\mathfrak{A}_{\text {sing. }}$. Then $z=\phi(z-b) \in \mathfrak{A}_{\text {sing }}$ if $z-b \in \mathfrak{A}_{\text {sing. It }}$ follows that if $z \in \mathfrak{A}_{\mathrm{inv}}$, then $z-b \in \mathfrak{A}_{\mathrm{inv}}$. From what we have proved above, $-b \in \mathfrak{A}_{\text {rad }}$. Thus $b=0$ for the stated choices of $T$ when $\mathfrak{A}$ is semi-simple. (By 
what we have just shown, the radical of a complex Banach algebra $\mathfrak{A}$ with unit $e$ can be characterized as the set of $b$ in $\mathfrak{A}$ such that $e+b z$ is invertible for all invertible $z$ in $\mathfrak{A}$; for when $b$ satisfies that condition and $z$ is in $\mathfrak{A}_{\text {inv }}$, the identity $z+b=\left(e+b z^{-1}\right) z$ implies that $z+b$ is in $\left.\mathfrak{A}_{\text {inv }}.\right)$

Conversely, if $b \in \mathfrak{A}_{\text {rad }}$ and $z \in \mathfrak{A}_{\text {inv }}$, then $z+b \in \mathfrak{A}_{\text {inv }}$ by the previous identity. If $z \in \mathfrak{A}_{\mathrm{inv}}^{\mathrm{e}}$, then $z+t b \in \mathfrak{A}_{\mathrm{inv}}$ for each $t$ in $[0,1]$ since each $t b \in \mathfrak{A}_{\mathrm{rad}}$. Thus $z+b \in \mathfrak{A}_{\mathrm{inv}}^{\mathrm{e}}$. If $z+b \in \mathfrak{A}_{\mathrm{inv}}$, then $z=(z+b)-b \in \mathfrak{A}_{\text {inv }}$ since $-b \in \mathfrak{A}_{\mathrm{rad}}$. It follows that if $z \in \mathfrak{A}_{\text {sing }}$, then $z+b \in \mathfrak{A}_{\text {sing }}$. Hence $\phi$ maps each of the sets $\mathfrak{A}_{\text {inv }}, \mathfrak{A}_{\text {inv }}^{\mathrm{e}}$ and $\mathfrak{A}_{\text {sing }}$ into itself, where $\phi(z)=z+b$. It follows that $\phi$ maps $T$ onto itself when $T$ is any of $\mathfrak{A}_{\mathrm{inv}}, \mathfrak{A}_{\mathrm{inv}}^{\mathrm{e}}$ or $\mathfrak{A}_{\mathrm{sing}}$, since for each $z$ in $T, z-b \in T$ (as $-b \in \mathfrak{A}_{\mathrm{rad}}$ ) and $z=\phi(z-b)$. Thus if all mappings such as $\phi$ are linear, $b=0$ for all $b$ in $\mathfrak{A}_{\text {rad }}$, and $\mathfrak{A}$ is semi-simple.

If $T=\mathfrak{A}_{\text {qnil }}$, it is a theorem of J. Zemánek [12] that $\phi(T)=T$ if and only if $b \in \mathfrak{A}_{\mathrm{rad}}$. This completes the proof of Proposition 2.1.

To prove Proposition 2.2, we may suppose, as before, that $\phi(z)=z+b$. Assume, first, that $\phi(T) \subseteq T$. Given $\epsilon= \pm 1$ and a non-zero complex number $\lambda$, let $z$ be $\epsilon \bar{\lambda} I+b$. Then $z \in T$, so $\epsilon z^{*}+b \in T$. Thus $\lambda I+b+\epsilon b^{*}$ is invertible for all non-zero complex $\lambda$. It follows that the spectrum of $b+\epsilon b^{*}$ is $\{0\}$. Since both $b+b^{*}$ and $i\left(b-b^{*}\right)$ are self-adjoint, both are 0 . Therefore, $b=0$.

Suppose, next, that $\phi$ is an affine mapping of $\mathfrak{A}$ into $\mathcal{B}(\mathcal{H})$ which maps $\mathfrak{A} \backslash T$ onto itself. With $z$ in $\mathfrak{A} \backslash T,-z \in \mathfrak{A} \backslash T$ (otherwise, $-z \in T$ and $z=-(-z) \in T$ by assumption). Thus $-(\mathfrak{A} \backslash T)=\mathfrak{A} \backslash T$ and Lemma 2.3 applies with $\mathfrak{A} \backslash T$ in place of $T$. Again, we may assume that $\phi(z)=z+b$ (but that $\phi$ maps $\mathfrak{A} \backslash T$ just into itself). If $z \in T$, then $z-b \in T$ (otherwise, $z-b \in \mathfrak{A} \backslash T$ and $z=z-b+b \in \mathfrak{A} \backslash T$ ). From the preceding, $-b=0$. This completes the proof of Proposition 2.2.

In the proofs of Propositions 2.1 and 2.2 given above, the "onto" assumption is made for the purpose of reduction (with the aid of Lemma 2.3) to affine mappings $z \rightarrow z+b$ that are simple translations. After this reduction, the translation mappings are into; for such mappings, it is shown that $b=0$.

Corollary 2.4. If $a$ and $b$ are elements of a complex, unital, semi-simple Banach algebra (in particular, a $C^{*}$-algebra) $\mathfrak{A}$ such that $\operatorname{sp}(z+a) \subseteq \operatorname{sp}(z+b)$ for each $z$ in $\mathfrak{A}$, then $a=b$.

Proof. By assumption, for all $z$ in $\mathfrak{A}$,

$$
\operatorname{sp}(z-b+a) \subseteq \operatorname{sp}(z-b+b)=\operatorname{sp}(z) .
$$

Thus, if $z$ is invertible (that is, if $0 \notin \operatorname{sp}(z)$ ), then $0 \notin \operatorname{sp}(z-b+a)$ and $z+c$ is invertible, where $c=a-b$. The translation $z \rightarrow z+c$ maps $\mathfrak{A}_{\text {inv }}$ into $\mathfrak{A}_{\text {inv }}$, whence $c=0$ and $a=b$.

\section{LINEAR MAPPINGS}

The case where the mapping is linear is of basic importance. Even the situation in which our Banach algebra is the $\left(\mathrm{C}^{*}-\right)$ algebra $M_{n}(\mathbb{C})$ of all complex $n \times n$ matrices is instructive. A good account of results characterizing linear mappings of $M_{n}(\mathbb{C})$ that preserve certain properties of matrices can be found in [9]. The subject has a venerable history. In 1897, Frobenius [2] showed that a mapping that preserves the determinant is the composition of an automorphism or an anti-automorphism of $M_{n}(\mathbb{C})$ with a left multiplication by a matrix of determinant 1. 
We give an argument to show that if $\phi$ is a linear mapping of $M_{n}(\mathbb{C})$ into $M_{n}(\mathbb{C})$ that maps the set of invertible matrices onto itself, then $\phi$ is the composition of an automorphism or an anti-automorphism and a left multiplication by an invertible matrix (namely, $\phi(I)$ ). This result, in the stronger form that requires only that $\phi$ map the invertible matrices into the invertible matrices, can be read from Theorem 2.1 of [10]. Each $B$ in $M_{n}(\mathbb{C})$ is the sum of two invertible matrices (for example, $(B-\lambda I)+\lambda I$, where $\lambda$ is some non-zero complex number not in $\operatorname{sp}(B))$. Thus $\phi$ maps $M_{n}(\mathbb{C})$ onto $M_{n}(\mathbb{C})$. Since $M_{n}(\mathbb{C})$ is finite dimensional over $\mathbb{C}$ and $\phi$ is linear, $\phi$ is a linear isomorphism. Replacing $\phi$ by $\phi(I)^{-1} \phi$, we may assume that $\phi(I)=I$. In this case, $\phi(A)-\lambda I$ is invertible exactly when $A-\lambda I$ is invertible, whence $\operatorname{sp}(A)=\operatorname{sp}(\phi(A))$. If $A$ has $n$ distinct eigenvalues, then $\phi(A)$ has these same distinct eigenvalues. By transforming a basis of eigenvectors for $A$ onto one for $\phi(A)$, we construct a similarity of $A$ with $\phi(A)$. It follows, in particular, that $A$ and $\phi(A)$ have the same determinant in this case. Now, the set of $A$ with distinct eigenvalues is dense in $M_{n}(\mathbb{C})$. (Think of an arbitrary matrix in upper triangular form and "perturb" its diagonal slightly.) Since $\phi$ and the determinant are continuous mappings on $M_{n}(\mathbb{C}), B$ and $\phi(B)$ have the same determinant for all $B$. From the Frobenius theorem, $\phi$ is an automorphism or an anti-automorphism. (Recall that the given $\phi$ has been multiplied by $\phi(I)^{-1}$.)

Conjecture 3.1. Each linear isomorphism $\phi$ of a $C^{*}$-algebra $\mathfrak{A}$ onto a $C^{*}$-algebra $\mathfrak{B}$ that maps $\mathfrak{A}_{\mathrm{inv}}$ onto $\mathfrak{B}_{\mathrm{inv}}$ is the composition of a Jordan isomorphism of $\mathfrak{A}$ onto $\mathfrak{B}$ and left multiplication by the invertible element $\phi(I)$.

In [6], Jafarian and Sourour establish 3.1 when the $\mathrm{C}^{*}$-algebra is $\mathcal{B}(\mathcal{H})$. More generally, they prove the corresponding result for mappings between $\mathcal{B}(X)$ and $\mathcal{B}(Y)$, where $X$ and $Y$ are Banach spaces.

Of course, one works with $\phi(I)^{-1} \phi$ and assumes that $\phi(I)=I$. For this modified mapping, the task is to show that squares are preserved. It is probably important (certainly, useful) to prove the automatic (norm) continuity of the mapping, though this continuity might be assumed in a preliminary conjecture. An example of Russo [11] shows that the "onto" hypothesis is essential for each $\mathrm{C}^{*}$-algebra $\mathfrak{A}$ other than $\mathbb{C}$, where $\mathfrak{B}$ is taken to be the algebra of $2 \times 2$ matrices with entries from $\mathfrak{A}$.

The conjecture restricted to the case where $\mathfrak{A}$ and $\mathfrak{B}$ are von Neumann algebras - or, even, factors - is a key case of much interest. In this connection, we can make use of the (absolute value of the) determinant of [3] and conjecture an almost direct extension of the Frobenius result.

Conjecture 3.2. If $\mathcal{M}$ and $\mathcal{N}$ are factors of type $I_{1}$, a linear isomorphism $\phi$ of $\mathcal{M}$ onto $\mathcal{N}$ that maps the unit of $\mathcal{M}$ to that of $\mathcal{N}$ preserves determinants if and only if it is an (algebraic) isomorphism or an anti-isomorphism.

We prove some results that support these conjectures.

Proposition 3.3. If $\phi$ is a linear isomorphism of a $C^{*}$-algebra $\mathfrak{A}$ onto a $C^{*}$-algebra $\mathfrak{B}$ that preserves adjoints, maps $I$ onto $I$, and $\mathfrak{A}_{\mathrm{inv}}$ onto $\mathfrak{B}_{\mathrm{inv}}$, then $\phi$ is a Jordan isomorphism of $\mathfrak{A}$ onto $\mathfrak{B}$.

Proof. Since $A-\lambda I$ is invertible precisely when $\phi(A)-\lambda I$ is, it follows that $A$ and $\phi(A)$ have identical spectra. Thus $\phi(A) \geq 0$ precisely when $A \geq 0$, as $\phi(A)$ is self-adjoint precisely when $A$ is. (By assumption, $\phi(A)$ is self-adjoint when $A$ is. If $A$ is not self-adjoint, $A \neq A^{*}$; whence $\phi(A) \neq \phi\left(A^{*}\right)=\phi(A)^{*}$, and $\phi(A)$ is not self-adjoint.) 
Since $\phi$ is an order isomorphism, under the present assumptions, [7] applies, and $\phi$ is a Jordan * isomorphism of $\mathfrak{A}$ onto $\mathfrak{B}$.

In [1], a similar result is proved that permits into mappings but adds the assumption of positivity.

Proposition 3.4. If $\phi$ is a linear isomorphism of a $C^{*}$-algebra $\mathfrak{A}$ onto an abelian $C^{*}$-algebra $\mathfrak{B}$ and $\phi$ maps $\mathfrak{A}_{\mathrm{inv}}$ onto $\mathfrak{B}_{\mathrm{inv}}$, then $\phi$ is an isomorphism of $\mathfrak{A}$ onto $\mathfrak{B}$ composed with multiplication by $\phi(I)$, so that $\mathfrak{A}$ is abelian.

Proof. Replacing $\phi$ by $\phi(I)^{-1} \phi$, we may assume that $\phi(I)=I$. As noted previously, $A$ and $\phi(A)$ have identical spectra. With $A$ self-adjoint, $\operatorname{sp}(A)$ and, hence, $\operatorname{sp}(\phi(A))$ are real. Since $\mathfrak{B}$ is abelian, $\phi(A)$ is self-adjoint. Thus $\phi$ is adjoint preserving and Proposition 3.3 applies. It follows that $\phi$ is a Jordan * isomorphism of $\mathfrak{A}$ onto $\mathcal{B}$. Thus $\mathfrak{A}$ is abelian and $\phi$ is a ${ }^{*}$ isomorphism of $\mathfrak{A}$ onto $\mathfrak{B}$.

We introduce a notion of "spectral additivity" with the aid of which we show that central elements map to central elements under unit and spectrum-preserving mappings. In the definition that follows, the notation $\mathcal{S}_{1}+\mathcal{S}_{2}$, where $\mathcal{S}_{1}$ and $\mathcal{S}_{2}$ are subsets of $\mathbb{C}$, denotes the set $\left\{z_{1}+z_{2}: z_{1} \in \mathcal{S}_{1}, z_{2} \in \mathcal{S}_{2}\right\}$.

Definition 3.5. An element $A$ of a $\mathrm{C}^{*}$-algebra $\mathfrak{A}$ is said to be spectrally additive (in $\mathfrak{A})$ when $\operatorname{sp}(A+B) \subseteq \operatorname{sp}(A)+\operatorname{sp}(B)$ for each $B$ in $\mathfrak{A}$.

This same definition may be made for elements of a (unital) Banach algebra $\mathfrak{A}$ (over $\mathbb{C}$ ). This is done in [4], where the concept of spectral additivity is studied in the context of Banach algebras with the aid of (purely algebraic) commutator results for "Schurian algebras". It is proved there that $A$ is spectrally additive in $\mathfrak{A}$ if and only if $A T-T A$ lies in the radical of $\mathfrak{A}$ for each $T$ in $\mathfrak{A}$. In particular, if $\mathfrak{A}$ is semi-simple, as is the case when $\mathfrak{A}$ is a $\mathrm{C}^{*}$-algebra, then $A$ is spectrally additive if and only if it lies in the center of $\mathfrak{A}$.

If $\phi$ is a spectrum-preserving, linear isomorphism of one $\mathrm{C}^{*}$-algebra $\mathfrak{A}$ onto another $\mathfrak{B}$ and $A$ is spectrally additive in $\mathfrak{A}$, then

$$
\operatorname{sp}(\phi(A)+\phi(B))=\operatorname{sp}(A+B) \subseteq \operatorname{sp}(A)+\operatorname{sp}(B)=\operatorname{sp}(\phi(A))+\operatorname{sp}(\phi(B)),
$$

for each element $\phi(B)$ in $\mathfrak{B}$. Thus $\phi(A)$ is spectrally additive in $\mathfrak{B}$.

Theorem 3.6. If $\phi$ is a linear isomorphism of one $C^{*}$-algebra $\mathfrak{A}$ onto another $\mathfrak{B}$ that maps $\mathfrak{A}_{\mathrm{inv}}$ onto $\mathfrak{B}_{\mathrm{inv}}$ and the unit I of $\mathfrak{A}$ onto that of $\mathfrak{B}$, then $\phi$ maps the center of $\mathfrak{A}$ isomorphically onto the center of $\mathfrak{B}$.

Proof. As noted, $T$ and $\phi(T)$ have the same spectra. From [4], the spectrally additive elements form the centers of $\mathfrak{A}$ and $\mathfrak{B}$. It follows that $\phi$ maps the center of $\mathfrak{A}$ onto that of $\mathfrak{B}$ and, from Proposition 3.4, that this mapping between centers is an isomorphism.

\section{The CASE OF "INTO" MAPPINGS}

We next consider the case of affine mappings that take $\mathfrak{A}_{\text {inv }}$ into itself. These mappings need not be constant or linear even when $\mathfrak{A}$ is a commutative $\mathrm{C}^{*}$-algebra, as the mapping $\left(\begin{array}{ll}a & 0 \\ 0 & b\end{array}\right) \rightarrow\left(\begin{array}{ll}a & 0 \\ 0 & 0\end{array}\right)+\left(\begin{array}{ll}0 & 0 \\ 0 & 1\end{array}\right)$ shows.

Theorem 4.1. Let $\mathfrak{A}$ be a $C^{*}$-algebra acting on $\mathcal{H}$ and containing the identity operator $I$ on $\mathcal{H}$. Given $A_{1}, A_{2}$ and $B$ in $\mathfrak{A}$, let $\phi(Z)=A_{1} Z A_{2}+B$. Then 
(1) $\phi\left(\mathfrak{A}_{\mathrm{inv}}\right) \subseteq \mathfrak{A}_{\mathrm{inv}}$ if and only if $A_{1} A_{2}+B$ has an inverse $R$ in $\mathcal{B}(\mathcal{H})$ and $A_{2} R A_{1}$ $(=E)$ is a central projection in $\mathfrak{A}$.

(2) $\phi\left(\mathfrak{A}_{\text {sing }}\right) \subseteq \mathfrak{A}_{\text {sing }}$ and $\phi(\mathfrak{A}) \nsubseteq \subseteq \mathfrak{A}_{\text {sing }}$ if and only if $A_{1} A_{2}+B$ has an inverse $R$ in $\mathcal{B}(\mathcal{H})$ and $A_{2} R A_{1}=I$.

If $\phi(I)=I$, then $R$ is $I$ in both cases (1) and (2) so that $A_{2} A_{1}$ is the central projection $E$ in case (1) and $I$ in case (2). As $B=I-A_{1} A_{2}$, in case (1), we have that $A_{2} B=(I-E) A_{2}$ and $B A_{1}=A_{1}(I-E)$. In case (2), we have that $B A_{1}=A_{2} B=0$, and $B^{2}=B$. With these identities, a careful calculation shows that, in case (1),

$$
\phi(Z)^{-1}=\phi\left(Z^{-1} E+(2 I-Z)(I-E)\right)
$$

for all $Z$ in $\mathfrak{A}_{\text {inv }}$ and, in case $(2)$,

$$
\phi(Z W)=\phi(Z) \phi(W)
$$

for all $Z$ and $W$ in $\mathfrak{A}$. From Theorem 4.1 , if $\phi\left(\mathfrak{A}_{\text {sing }}\right) \subseteq \mathfrak{A}_{\text {sing }}$ and $\phi(\mathfrak{A}) \nsubseteq \mathfrak{A}_{\text {sing }}$, then $\phi\left(\mathfrak{A}_{\text {inv }}\right) \subseteq \mathfrak{A}_{\text {inv }}$. We have the following corollary of Theorem 4.1.

Corollary 4.2. Let $\mathfrak{A}$ be a $C^{*}$-algebra acting on $\mathcal{H}$ and containing the identity operator $I$ on $\mathcal{H}$. With $A$ and $B$ in $\mathcal{B}(\mathcal{H})$, let $\phi(Z)=A Z+B$. Then

(1) $\phi\left(\mathfrak{A}_{\mathrm{inv}}\right) \subseteq \mathfrak{A}_{\mathrm{inv}}$ if and only if there is a central projection $E$ in $\mathfrak{A}$ and an $R^{-1}$ in $\mathfrak{A}_{\text {inv }}$ such that $A=R^{-1} E$ and $B=R^{-1}(I-E)$.

(2) If $\mathfrak{A} \neq \mathbb{C} I$, then $\phi\left(\mathfrak{A}_{\text {sing }}\right) \subseteq \mathfrak{A}_{\text {sing }}$ and $\phi(\mathfrak{A}) \nsubseteq \mathfrak{A}_{\text {sing }}$ if and only if $A \in \mathfrak{A}_{\text {inv }}$ and $B=0$.

Proof. In both cases (1) and (2) of the corollary, the "umbrella" hypothesis of Theorem 4.1 - that $A$ and $B$ are in $\mathfrak{A}$ (as opposed to just $\mathcal{B}(\mathcal{H})$ ) - are satisfied. In case (1), if $E$ and $R$ are as described, then $A$ and $B$ are in $\mathfrak{A}$. On the other hand, if $\phi\left(\mathfrak{A}_{\text {inv }}\right) \subseteq \mathfrak{A}_{\text {inv }}$, then $A I+B(=\phi(I))$ and $A(-I)+B(=\phi(-I))$ are in $\mathfrak{A}_{\text {inv. }}$. Thus $A\left(=\frac{1}{2}[\phi(I)-\phi(-I)]\right)$ and $B\left(=\frac{1}{2}[\phi(I)+\phi(-I)]\right)$ are in $\mathfrak{A}$.

In case $(2), B=A(0)+B=\phi(0) \in \mathfrak{A}_{\text {sing }}$, whence $B \in \mathfrak{A}$. At the same time, if $H=H^{*} \in \mathfrak{A}$ and $\lambda \in \operatorname{sp}(H)$, then $A(H-\lambda I)+B=\phi(H-\lambda I) \in \mathfrak{A}_{\text {sing. Thus }}$ $A(H-\lambda I) \in \mathfrak{A}$. Since $\mathfrak{A}$ is not $\mathbb{C} I$, there is some self-adjoint $H$ in $\mathfrak{A}$ with at least two distinct spectral values $\lambda_{1}$ and $\lambda_{2}$. As $\left(\lambda_{2}-\lambda_{1}\right) A=A\left(H-\lambda_{1} I\right)-A\left(H-\lambda_{2} I\right) \in \mathfrak{A}$, $A \in \mathfrak{A}$.

It follows that Theorem 4.1 is applicable in cases (1) and (2).

(1) From Theorem 4.1, $\phi\left(\mathfrak{A}_{\text {inv }}\right) \subseteq \mathfrak{A}_{\text {inv }}$ if and only if $A+B$ has an inverse $R$ in $\mathcal{B}(\mathcal{H})$ and $R A$ is a central projection $E$ in $\mathfrak{A}$. If $\phi\left(\mathfrak{A}_{\text {inv }}\right) \subseteq \mathfrak{A}_{\text {inv }}$, then $R^{-1}=$ $A+B=\phi(I) \in \mathfrak{A}_{\mathrm{inv}}, A=R^{-1} E$, and $B=R^{-1}-A=R^{-1}(I-E)$. Conversely, if $R$ and $E$ are as described, then $R A$ is the central projection $E$ and $A+B\left(=R^{-1}\right)$ has an inverse $R$ in $\mathcal{B}(\mathcal{H})$. Thus $\phi\left(\mathfrak{A}_{\text {inv }}\right) \subseteq \mathfrak{A}_{\text {inv }}$.

(2) From Theorem 4.1, $\phi\left(\mathfrak{A}_{\text {sing }}\right) \subseteq \mathfrak{A}_{\text {sing }}$ and $\phi(\mathfrak{A}) \nsubseteq \mathfrak{A}_{\text {sing }}$ if and only if $A+B$ has an inverse $R$ in $\mathcal{B}(\mathcal{H})$ and $R A=I$. Again, $R$ is necessarily in $\mathfrak{A}$. Of course, $R$ is as described in (2) of Theorem 4.1 if and only if $A \in \mathfrak{A}_{\text {inv }}, R=A^{-1}$, and $B=R^{-1}-A=0$.

The conclusions of (2) in Corollary 4.2 do not hold when $\mathfrak{A}$ is $\mathbb{C} I$ (choose $A$ not a scalar and 0 for $B$, in that case). The conclusions of (1) in Corollary 4.2 do not necessarily hold when it is assumed only that $\phi\left(\mathfrak{A}_{\text {inv }}\right) \subseteq \mathcal{B}(\mathcal{H})_{\text {inv }}$. For example, let $\mathfrak{A}$ be $\left\{\left(\begin{array}{cc}z & 0 \\ 0 & z\end{array}\right): z \in \mathbb{C}\right\}, A$ be $\left(\begin{array}{ll}0 & 0 \\ 1 & 0\end{array}\right)$, and $B$ be $I-A$. Then $A Z+B$ is invertible for all $Z$ in $\mathfrak{A}$. If $A=R E$ and $B=R(I-E)$ for a projection $E$ (necessarily, non-zero) 
and any $R$, then $R=A+B=I$ and $A=E$. But then $E=E^{2}=A^{2}=0 \neq E$, a contradiction.

Proof of Theorem 4.1. We first note that if $\phi(I)$ is invertible, then $\phi(Z)$ is invertible if and only if $\psi(Z)=E Z+F$ is invertible, where $E=A_{2} R A_{1}, R=\phi(I)^{-1}$ and $F=I-E$. Indeed, since $R\left(A_{1} A_{2}+B\right)=I$,

$$
R \phi(Z)=R A_{1} Z A_{2}+R B=I+R A_{1}(Z-I) A_{2}
$$

while

$$
\psi(Z)=E Z+I-E=I+A_{2} R A_{1}(Z-I) .
$$

Thus $R \phi(Z)$ (and, hence, $\phi(Z)$ ) is invertible if and only if $\psi(Z)$ is invertible by the result that $e+x y$ is invertible if and only if $e+y x$ is invertible, where $x$ and $y$ are elements of a ring with identity $e$. (See [8, Proposition 3.2.8]. The formula for $\phi(Z)^{-1}$ given after Theorem 4.1 can be derived from the identity $(e+y x)^{-1}=$ $e-y(e+x y)^{-1} x$ and the formula for $\psi(Z)^{-1}$ given below.)

(1) Suppose $\phi(I)$ is invertible and $E$ is a central projection in $\mathfrak{A}$. If $Z \in \mathfrak{A}_{\text {inv }}$, then $\psi(Z)^{-1}=\psi\left(Z^{-1}\right)$. Thus $\psi(Z) \in \mathfrak{A}_{\text {inv }}$, so $\phi(Z) \in \mathfrak{A}_{\text {inv }}$. It follows that $\phi\left(\mathfrak{A}_{\text {inv }}\right) \subseteq \mathfrak{A}_{\text {inv }}$.

Suppose $\phi\left(\mathfrak{A}_{\text {inv }}\right) \subseteq \mathfrak{A}_{\text {inv }}$. Then $\psi\left(\mathfrak{A}_{\text {inv }}\right) \subseteq \mathfrak{A}_{\text {inv }}$. Given $C$ in $\mathfrak{A}$, if $\lambda \notin \operatorname{sp}(C)$ and $\lambda \neq 0$, then $Z=I-\lambda^{-1} C$ is in $\mathfrak{A}_{\mathrm{inv}}$ and hence so is $\psi(Z)=E Z+I-E=I-\lambda^{-1} E C$; consequently, $\lambda \notin \operatorname{sp}(E C)$. Thus,

$$
\operatorname{sp}(E C) \subseteq \operatorname{sp}(C) \cup\{0\}
$$

for all $C \in \mathfrak{A}$. From $(*)$, with $I$ for $C$, we have $\operatorname{sp}(E) \subseteq\{0,1\}$. Thus $\operatorname{sp}\left(E^{*}\right) \subseteq$ $\{0,1\}$. With $E^{*}$ for $C$ in $(*)$, we have $\operatorname{sp}\left(E E^{*}\right) \subseteq\{0,1\}$. Thus $E E^{*}$ is a projection, so $E$ is a partial isometry, and $E E^{*} E=E$. Choosing $\left(I-E^{*}\right)^{2}$ for $C$ in $(*)$ and applying [8, Propositions 3.2.8, 3.2.10], we have that

$$
\operatorname{sp}\left(\left(I-E^{*}\right) E\left(I-E^{*}\right)\right) \subseteq \operatorname{sp}\left(E\left(I-E^{*}\right)^{2}\right) \cup\{0\} \subseteq\{0,1\} .
$$

Now, since $E=E E^{*} E$,

$$
\left(E-E E^{*}\right)^{*}\left(E-E E^{*}\right)=\left(E^{*}-E E^{*}\right) E\left(I-E^{*}\right)=-\left(I-E^{*}\right) E\left(I-E^{*}\right) .
$$

It follows that the spectrum of $-\left(I-E^{*}\right) E\left(I-E^{*}\right)$ consists of non-negative real numbers and hence this spectrum is $\{0\}$ by $(* *)$. Therefore, $E-E E^{*}=0$, and $E$ is a projection.

With $H$ self-adjoint in $\mathfrak{A}_{\text {inv }}$, define $\psi_{1}(Z)=\psi(H)^{-1} \psi(H Z)$. Then $\psi_{1}\left(\mathfrak{A}_{\text {inv }}\right) \subseteq$ $\mathfrak{A}_{\text {inv }}$ and $\psi_{1}(I)=I$. Now, $\psi_{1}(Z)=(E H+F)^{-1} E H Z+(E H+F)^{-1} F$. Applying what we have proved to this point to $\psi_{1}$, we have that $(E H+F)^{-1} E H$ is a projection. In particular, $(E H+F)^{-1} E H$ is self-adjoint. Then

$$
(E H+F)(E H+F)^{-1} E H(E H+F)^{*}=E H(E H)^{*}+E H F
$$

is self-adjoint and hence so is $E H F$. Thus $E H F=F H E$, and $E H F=E E H F=$ $E F H E=0$. It follows that

$$
E H=E H E=(E H E)^{*}=(E H)^{*}=H E .
$$

For an arbitrary self-adjoint $A$ in $\mathfrak{A}$, we have that $(\|A\|+1) I-A$ is self-adjoint and invertible in $\mathfrak{A}$. Thus it commutes with $E$ as does $A$. Since $E$ commutes with each self-adjoint element of $\mathfrak{A}, E$ lies in the center of $\mathfrak{A}$. 
(2) If $A_{2} R A_{1}=I$, then $\psi(Z)=Z$. As noted in the discussion preceding the proof of (1), $\phi(Z) \in \mathfrak{A}_{\text {sing }}$ if and only if $\psi(Z) \in \mathfrak{A}_{\text {sing }}$. Thus, under the present assumption, $\phi\left(\mathfrak{A}_{\text {sing }}\right) \subseteq \mathfrak{A}_{\text {sing }}$ and $\phi(\mathfrak{A}) \nsubseteq \mathfrak{A}_{\text {sing }}$.

Suppose that $\phi\left(\mathfrak{A}_{\text {sing }}\right) \subseteq \mathfrak{A}_{\text {sing }}$ and $\phi(I)$ is invertible. Then $\psi\left(\mathfrak{A}_{\text {sing }}\right) \subseteq \mathfrak{A}_{\text {sing }}$. Given $C \in \mathfrak{A}$, if $\lambda \in \operatorname{sp}(C)$ and $\lambda \neq 0$, then $Z=I-\lambda^{-1} C$ is in $\mathfrak{A}_{\text {sing }}$ and hence so is $\psi(Z)=E Z+I-E=I-\lambda^{-1} E C$; consequently, $\lambda \in \operatorname{sp}(E C)$. Hence, $\operatorname{sp}(C) \subseteq \operatorname{sp}(E C) \cup\{0\}$ for all $C$ in $\mathfrak{A}$. With $E^{*}$ in place of $C$, we see that $\operatorname{sp}\left(E^{*}\right)$, and hence $\operatorname{sp}(E)$, consists of non-negative real numbers. Suppose that $E$ is not invertible. Then

$$
1 \in \operatorname{sp}(I-E) \subseteq \operatorname{sp}(E(I-E)) \cup\{0\} .
$$

Hence there is a $\lambda$ in $\operatorname{sp}(E)$ such that $1=\lambda-\lambda^{2}$. There is no such real $\lambda-$ contradicting our information about $\operatorname{sp}(E)$. Thus $E$ is invertible. Since $Z+F=$ $\psi\left(E^{-1} Z\right) \in \mathfrak{A}_{\text {sing }}$ when $Z \in \mathfrak{A}_{\text {sing }}$, it follows from the comments preceding Corollary 2.4 that $F \in \mathfrak{A}_{\text {rad }}(=\{0\})$; whence $F=0$ and $E=I$.

We show that the assumption that $\phi(I)$ is invertible is fulfilled. By hypothesis, $\phi\left(Z_{0}\right)$ is invertible for some $Z_{0}$ in $\mathfrak{A}$. Then $Z_{0}$ is invertible, since $\phi\left(\mathfrak{A}_{\text {sing }}\right) \subseteq \mathfrak{A}_{\text {sing }}$. Define $\phi_{1}(Z)=\phi\left(Z_{0} Z\right)$. Then $\phi_{1}\left(\mathfrak{A}_{\text {sing }}\right) \subseteq \mathfrak{A}_{\text {sing. }}$. As $\phi_{1}(I)=\phi\left(Z_{0}\right)$ is invertible, from what we have proved, $A_{1} Z_{0} A_{2}+B$ has an inverse $R_{1}$ for which $A_{2} R_{1} A_{1} Z_{0}=I$. From (1) of this theorem, $\phi_{1}\left(\mathfrak{A}_{\text {inv }}\right) \subseteq \mathfrak{A}_{\text {inv }}$. In particular, $\phi(I)=\phi_{1}\left(Z_{0}^{-1}\right)$ is invertible.

\section{REFERENCES}

1. M-D. Choi, D. Hadwin, E. Nordgren, H. Radjavi, and P. Rosenthal, On positive linear maps preserving invertibility, J. Functional Anal. 59 (1984), 462-469. MR 86a:46071

2. G. Frobenius, Über die Darstellung der endlichen Gruppen durch lineare Substitutionen, I. Sitzungberichte der Königlich Preussischen Akademie der Wissenschaften zu Berlin, 1897, pp. 994-1015.

3. B. Fuglede and R. Kadison, Determinant theory in finite factors, Ann. of Math. 55 (1952), 520-530. MR 14:660a

4. L. Harris and R. Kadison, Schurian algebras and spectral additivity, J. of Algebra 180 (1996), $175-186$.

5. E. Hille and R. Phillips, Functional Analysis and Semi-Groups, AMS, Providence, 1957. MR 54:11077

6. A. Jafarian and A. Sourour, Spectrum-preserving linear maps, J. Functional Anal. 66 (1986), 255-261. MR 87m:47011

7. R. Kadison, A generalized Schwarz inequality and algebraic invariants for operator algebras, Ann. of Math. 56 (1952), 493-502. MR 14:481c

8. R. Kadison and J. Ringrose, Fundamentals of the Theory of Operator Algebras, Academic Press, Orlando, Vol. I, 1983, Vol. II, 1986. MR 88d:46106

9. M. Marcus, Linear transformations on matrices, J. Res. Natl. Bur. Stand. 75B (1971), 107113. MR 46:9056

10. M. Marcus and R. Purves, Linear transformations on algebras of matrices: The invariance of the elementary symmetric functions, Canadian J. Math. 11 (1959), 383-396. MR 21:4167

11. B. Russo, Linear mappings of operator algebras, Proc. Amer. Math. Soc. 17 (1966), 10191022. MR 33:6428

12. J. Zemánek, Concerning spectral characterizations of the radical in Banach algebras, Comment. Math. Univ. Carolinæ17 (4) (1976), 689-691. MR 55:1070

Department of Mathematics, University of Kentucky, Lexington, Kentucky 405060027

Department of Mathematics, University of Pennsylvania, Philadelphia, Pennsylvania $19104-6395$ 